Proceeding Series of the Brazilian Society of Computational and Applied Mathematics

\title{
Uso de transformada wavelet discreta ortogonal e gráfico de recorrência para caracterização de sistemas dinâmicos não-lineares
}

\section{Barbara Maximino da Fonseca Reis ${ }^{1}$}

Programa de Pós-Graduação em Computação Aplicada, INPE, São José dos Campos, SP

Elbert E. N. Macau ${ }^{2}$

Laboratório Associado de Computação e Matemática Aplicada, INPE, São José dos Campos, SP Marcos Gonçalves Quiles ${ }^{3}$

Instituto de Ciência e Tecnologia, Unifesp, São José dos Campos, SP

Margarete Oliveira Domingues ${ }^{4}$

Laboratório Associado de Computação e Matemática Aplicada, INPE, São José dos Campos, SP

Resumo. Um sistema dinâmico possui invariantes que são obtidos por meio do gráfico de recorrência e suas medidas de quantificação. Para reduzir a complexidade computacional, este trabalho propõe utilizar a transformada wavelet discreta ortogonal para gerar um sinal suavizado que preserve a dinâmica do sistema.

Palavras-chave. Sistema Dinâmico, Gráfico de Recorrência, Análise de Quantificação de Recorrência, Transformada Wavelet Discreta Ortogonal, Mapa Logístico.

\section{Introdução}

A compreensão do mundo físico depende de observações, medidas, análise e predição de padrões expressos na natureza. Grande quantidade de sistemas possuem dinâmicas complexas, não-lineares e não-estacionárias, que dificultam a compreensão efetiva do sistema. Para tentar compreende-las, utilizam-se métodos como cálculo de dimensão fractal, expoente de Lyapunov e entropia [4]. Porém, tais métodos possuem desvantagens, como a necessidade de séries temporais longas, alteração dos resultados devido a presença de ruídos, dificuldade para analisar sistemas não-estacionários [7].

Nas últimas décadas, os gráficos de recorrência [4] tornaram-se uma alternativa para o estudo de sistemas complexos. Um gráfico de recorrência é uma matriz binária simétrica

\footnotetext{
${ }^{1}$ barbara.reis@inpe.br

${ }^{2}$ elbert.macau@inpe.br

${ }^{3}$ quiles@unifesp.br

${ }^{4}$ margarete.domingues@inpe.br
} 
que demonstra a proximidade de dois estados diferentes no espaço de fases a partir de uma determinada definição de proximidade. Desta forma, o gráfico de recorrência permite tanto visualizar as estruturas da série temporal quanto estimar invariantes presentes no sistema. Para extrair as informações presentes no gráfico de recorrência, Webber e Zbilut [10] desenvolveram a análise de quantificação de recorrência. Esta análise gera quantificações estatísticas baseadas na ocorrência de pontos e de linhas, diagonais e verticais, no gráfico de recorrência.

Contudo, a quantidade de pontos de um gráfico de recorrência pode ser alta, demandando um alto custo computacional para calcular as medidas de quantificação. Para resolver este problema, buscam-se métodos que diminuam a quantidade de pontos e preservem as invariantes do sistema. Yan et al. [11], Antoniou e Vorlow [1] propõem utilizar a transformada wavelet para suavizar o sinal enquanto Costa [2] propõem um método de suavização do gráfico de recorrência. Baseando-se na ideia de suavização do sinal, este trabalho propõe aplicar a transformada wavelet discreta ortogonal para suavizar o sinal e, assim, verificar se as dinâmicas do sistema são preservadas por meio da análise de quantificação de recorrência.

\section{Fundamentos teóricos}

Para a realização deste trabalho, foram utilizadas as técnicas: transformada wavelet discreta, gráfico de recorrência e análise de quantificação de recorrência.

\subsection{Transformada wavelet discreta}

A transformada wavelet é uma transformação integral e linear que utiliza como base uma função wavelet analisadora escolhida com certas características de localização, normalização e momentos [6].

Com essas propriedades satisfeitas essa transformada permite analisar, por exemplo, sinais em multiescalas, estruturas relacionadas a ruídos brancos não-lineares e compactação das informações [5].

Em particular, neste trabalho utiliza-se a transformada wavelet discreta ortogonal de Daubechies de ordem 1 [3] com o intuito de obter-se uma versão compacta do sinal analisado de forma a manter a dinâmica que ele representa.

\subsection{Gráfico de recorrência e análise de quantificação de recorrência}

O teorema de recorrência de Poincaré [9] afirma que, depois de um certo tempo, alguns sistemas retornarão a um estado muito próximo do estado inicial. Baseado neste teorema, Eckmann et al. [4] criaram o gráfico de recorrência $(\mathrm{R})$ para visualizar a dinâmica de sistemas recorrentes. Matematicamente, o gráfico de recorrência é gerado por:

$$
R_{i, j}^{m, \rho}=\theta\left(\rho-\left\|x_{i}-x_{j}\right\|\right)
$$


com $x_{i}, x_{j} \in \mathbb{R}^{m}, i, j=1, \ldots, N$. Nesta representação, $N$ é o número de estados, $\rho$ é raio de vizinhança no ponto $x_{i},\|$.$\| é a norma de vizinhança, \theta($.$) é função de Heaviside e m$ é a dimensão de imersão.

Se $R_{i, j}=1$, o estado é dito recorrente e um ponto preto é marcado no gráfico. Se $R_{i, j}=0$, o estado é dito não recorrente e um ponto branco é marcado no gráfico de recorrência.

Devido a dificuldade de classificação visual dos gráficos de recorrência, Webber e Zbilut [10] criaram a análise de quantificação de recorrência (RQA) como uma forma de potencializar estas classificações. RQA é uma técnica não-linear capaz de quantificar a dinâmica e a previsibilidade de um sistema dinâmico a partir de estruturas presentes no gráfico de recorrência, ou seja, a RQA utiliza a densidade de pontos de recorrência e as estruturas de linhas diagonais e verticais do gráfico para calcular as quantificações [7]. A Tabela 1 apresenta as quantificações utilizadas neste trabalho.

Tabela 1: Medidas de quantificação de recorrência.

\begin{tabular}{|l|l|l|}
\hline Medidas & Sigla & Descrição \\
\hline Taxa de recorrência & RR & $\begin{array}{l}\text { Mede a densidade dos pontos de recorrência no } \\
\text { gráfico de recorrência. }\end{array}$ \\
\hline Determinismo & DET & $\begin{array}{l}\text { Razão entre o número de pontos de recorrência que } \\
\text { formam as estruturas diagonais e todos os pontos de } \\
\text { recorrência. }\end{array}$ \\
\hline $\begin{array}{l}\text { Comprimento máximo } \\
\text { das linhas diagonais }\end{array}$ & $\mathrm{L}_{m a x}$ & $\begin{array}{l}\text { Indica o tempo máximo em que dois segmentos de } \\
\text { uma trajetória ficaram próximos um do outro. }\end{array}$ \\
\hline Entropia & ENTR & $\begin{array}{l}\text { Representa a distribuição de frequências dos compri- } \\
\text { mentos das linhas diagonais e reflete a complexidade } \\
\text { da estrutura determinística presente no sistema. }\end{array}$ \\
\hline
\end{tabular}

\section{Resultados e discussão}

Em relação aos sistemas dinâmicos, um mapa unidimensional é o modelo mais simples para a investigação de qualquer ferramenta. Devido a sua simplicidade, o mapa logístico foi utilizado como um teste inicial.

Popularizado por May [8], o mapa logístico é um modelo populacional para insetos descrito por:

$$
x_{n+1}=r x_{n}\left(1-x_{n}\right),
$$

no qual $x_{n}$ representa o número de indivíduos na $n$-ésima geração e $r$ é o parâmetro de controle que age como uma normalização entre a taxa de crescimento e a taxa de mortalidade da população.

Obtém-se a série temporal do mapa logístico fixando o valor do parâmetro de controle $r$ e iterando recursivamente o mapa a partir de uma condição inicial $x_{0}$. Nas primeiras 
iterações da série, geram-se valores aleatórios para $x_{n}$, denominados transientes. Após o período transiente, os valores de $x_{n}$ podem ficar alternando entre $N$ valores fixos em uma série periódica ou entre valores de uma série aperiódica que caracterizam um comportamento caótico.

Portanto, gerou-se 240 trajetórias, cada uma com 2048 pontos (excluindo os 512 transientes), que representassem os diversos tipos de dinâmicas presentes no mapa logístico quando o parâmetro de controle varia entre $2.8 \leq r \leq 4.0$.

Em seguida, obteve-se o gráfico de recorrência (com o valor limiar $\rho=0.10$ ) e calculouse as medidas de quantificação de recorrência de cada uma dessas trajetórias. A Figura 1 apresenta os resultados obtidos. O diagrama de bifurcação e o expoente de Lyapunov foram utilizados na identificação do tipo de dinâmica do sistema. Observa-se que, diferentemente das trajetórias caóticas, as órbitas periódicas aparecem caracterizadas como tendo alto grau de determinismo e alto valor de tamanho da maior diagonal, indicando um comportamento regular.

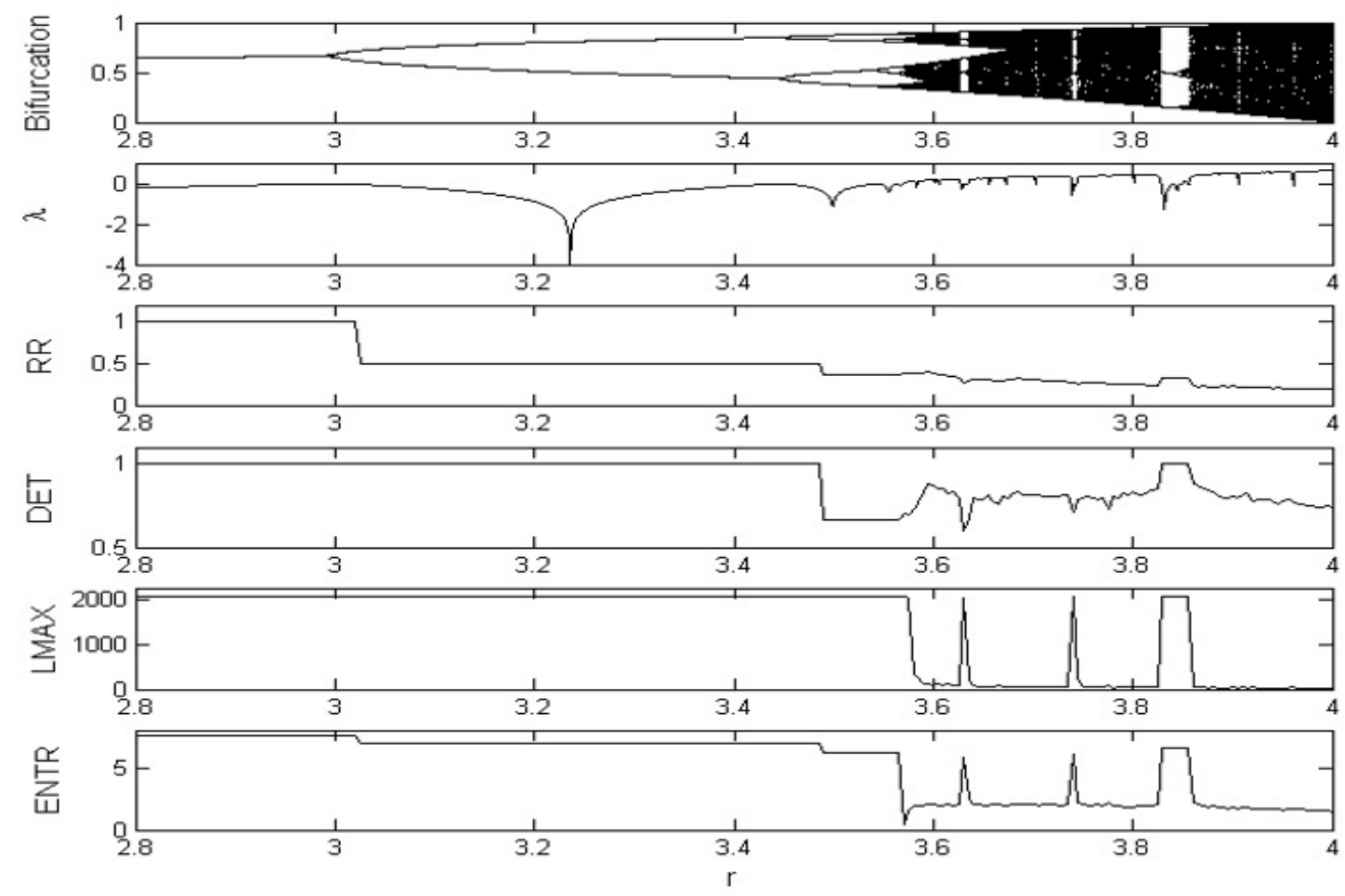

Figura 1: RQA para o mapa logístico.

Neste trabalho, utilizou-se a transformada wavelet discreta ortogonal para reduzir o tamanho das séries temporais e, assim, preservar a essência destas séries. Para isso, aplicou-se a transformada wavelet discreta ortogonal de Daubechies de ordem 1, com um nível de resolução, para obter séries suavizadas com a metade da quantidade de pontos das séries originais. Em seguida, gerou-se o gráfico de recorrência e calculou-se as medidas 
de quantificação de cada trajetória (Figura 2). Observa-se que, mesmo utilizando uma versão suavizada do sinal original, as medidas determinismo, tamanho da maior diagonal e entropia detectaram as mudanças na dinâmica do sistema.

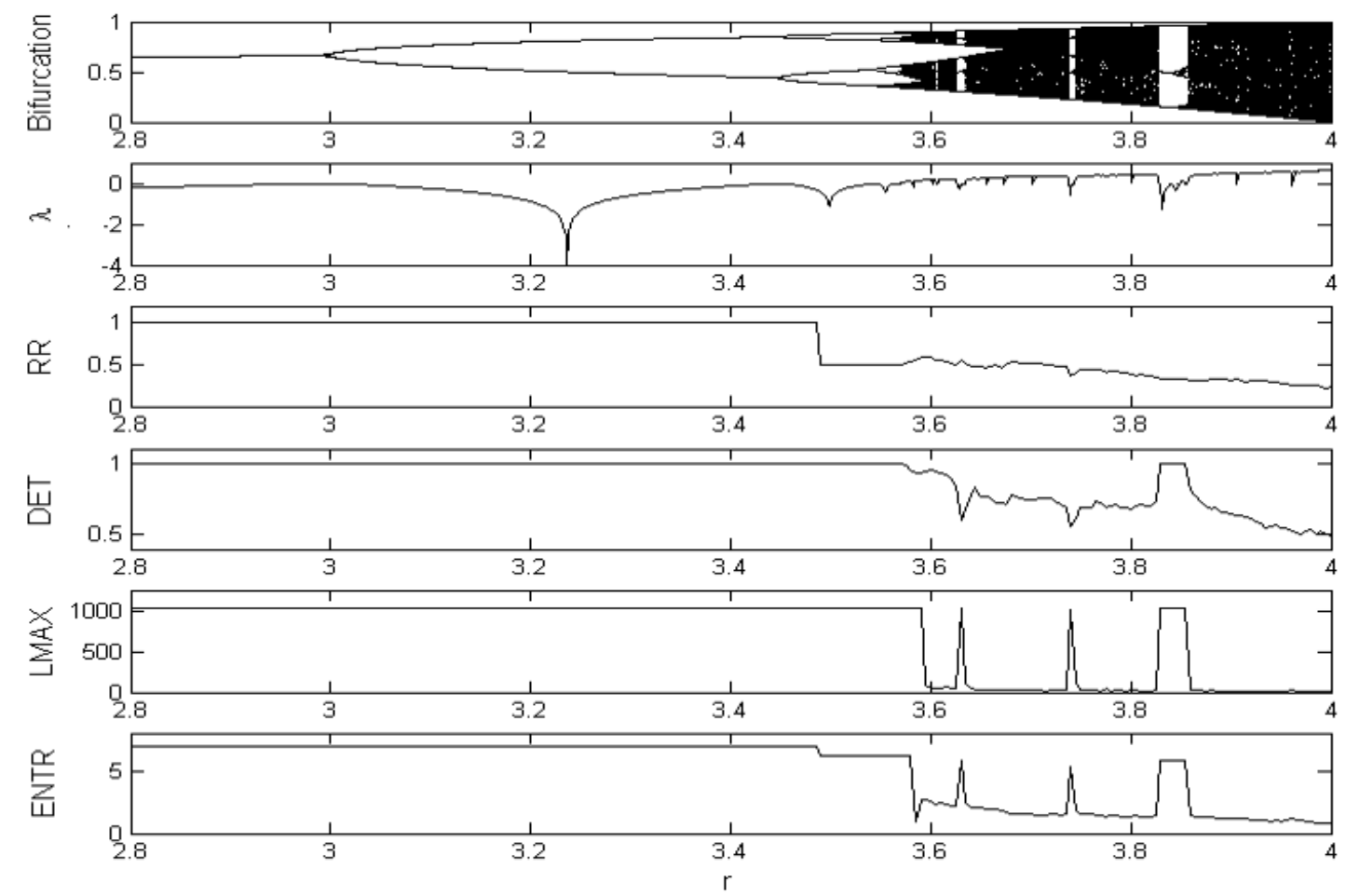

Figura 2: RQA dos gráficos de recorrência das séries resultantes da aplicação da transformada wavelet discreta ortogonal com um nível de resolução.

Aplicando a transformada wavelet discreta ortogonal de Daubechies de ordem 1, com dois níveis de resolução, obteve-se séries suavizadas com um quarto do tamanho das trajetórias originais. Novamente, após aplicar a transformada, gerou-se os gráficos de recorrência e calculou-se as medidas de quantificação de cada série. Como pode ser observado na Figura 3, as medidas determinismo, tamanho da maior diagonal e entropia continuaram detectando as mudanças na dinâmica do sistema.

\section{Conclusões e futuras perspectivas}

Para o mapa logístico, a transformada wavelet discreta ortogonal foi capaz de gerar séries suavizadas que preservaram as dinâmicas do sistema. Neste caso, as medidas determinismo, tamanho da maior diagonal e entropia foram capazes de localizar as regiões estáveis do sistema.

Como trabalho futuro, visa-se utilizar outras funções wavelet para decompor o sinal assim como calcular outras medidas de quantificações. Além disso, espera-se aplicar esta 

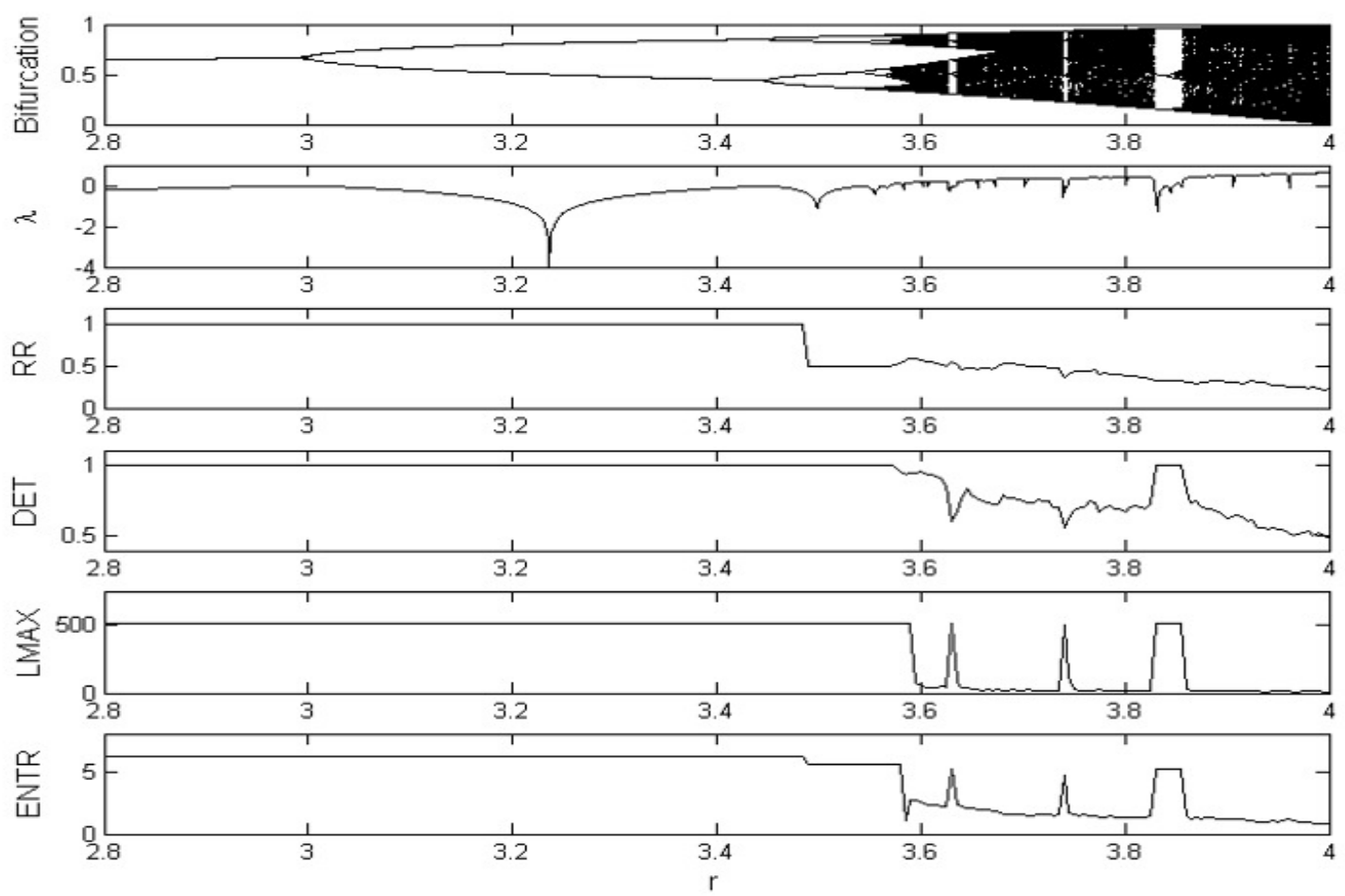

Figura 3: RQA dos gráficos de recorrência das séries resultantes da aplicação da transformada wavelet discreta ortogonal de Daubechies de ordem 1 com dois níveis de resolução.

abordagem em séries provenientes de sistemas reais.

\section{Agradecimentos}

Agradecimentos à Capes, à FAPESP e ao CNPq pelo apoio financeiro.

\section{Referências}

[1] A. Antoniou and C. E. Vorlow, Recurrence quantification analysis of wavelet prefiltered index returns, Physica A: Statistical Mechanics and its Applications, vol. $344,257-262$, (2004).

[2] D. G. B. Costa, Uso de gráfico de recorrência e redes complexas para caracterização de sistemas complexos de baixa dimensão, Dissertação de Mestrado em Computação Aplicada, INPE, (2014).

[3] I. Daubechies, Ten Lectures on Wavelets, Society for Industrial and Applied Mathematics, (1992). 
[4] J. Eckmann, S. Kamphorst and D. Ruelle, Recurrence plots of dynamical systems, Europhysics Letters, 973-977, (1987).

[5] B. B. Hubbard, The World According to Wavelets: The Story of a Mathematical Technique in the Making, Second Edition, A K Peters, (1998).

[6] S. G. Mallat, A Wavelet Tour of Signal Processing, Academic Press, (1999).

[7] N. Marwan, M. C. Romano, M. Thiel and J. Kurths, Recurrence plots for the analysis of complex systems, Physics Reports, vol. 438, 237-329, (2007).

[8] R. M. May, Simple mathematical models with very complicated dynamics, Nature, vol. 261, 459-467, (1976).

[9] H. Poincaré, Sur le problème des trois corps et les équations de la dynamique, Acta mathematica, vol. 13, 1-270, (1890).

[10] C. L. Webber Jr and J. P. Zbilut, Dynamical assessment of physiological systems and states using recurrence plot strategies, Journal of Applied Physiology, vol. 76, 965-973, (1994).

[11] J. Yan, C. Zhou, C. Xia, Y. Wang, F. Li, R. Guo and H. Yan, Recurrence quantification analysis base on wavelet packets for wrist pulse, 2010 3rd International Conference on Biomedical Engineering and Informatics, vol. 3, 1011-1015, (2010). 